\title{
Effect of static magnetic field on erythrocytes characterizations
}

\author{
Mohamed A. Elblbesy \\ Medical Biophysics Department, Medical Research Institute, Alexandria University, Alexandria, Egypt. \\ Email: mimizizo@yahoo.com
}

Received 29 November 2009; revised 15 December 2009; accepted 28 December 2009.

\begin{abstract}
The interaction of static magnetic field (SMF) with living organisms is a rapidly growing field of investigation. Recently, exposure to moderate intensity SMFs (1 mT - $1 \mathrm{~T})$ has attracted much attention for its various medical applications. This study was designed to show the microscopic effect of SMF on erythrocytes in vitro. For this purpose SMF system was constructed in my lab on basis of the idea of cell tracking velocimetry system. The changes in erythrocytes surface area, sphericity, and adhesion number for erythrocytes were calculated to quantify the effect of SMF on erythrocytes characterizations. The results showed that SMF increased erythrocytes surface area and reduced their sphericity. The adhesion number of erythrocytes under the influence of SMF showed the tendency of erythrocytes to adhere with each other. These findings indicate that more study on microscopic scales must be carried out in order to investigate the effect of SMF on erythrocytes.
\end{abstract}

Keywords: Static Magnetic Field; Erythrocytes; Surface Area; Shericity, Adhesion

\section{INTRODUCTION}

During the last few years there has been an increasing interest in the influence of magnetic field on biological cell [1]. Many attempts have been made to measure the magnetic effects. Historically, there are several significant studies in the field of erythrocyte rheology [2].

Reports from in vitro research indicate that low level high frequency fields may alter membrane structural and functional properties that trigger cellular responses. It was hypothesized that the cell membrane may be susceptible to low level high frequency fields, especially when these fields are amplitude modulated at extremely low frequencies [3]. There are abundant data on the effects of weak physical signals on cell functional activity. However cell targets for these signals are not established yet. Since water is the main component of biological systems and all metabolic processes take place in aqueous medium it was supposed that even minor changes in the physico-chemical properties of water could significant modify cell functional activity. The data about the effect of EMF on water specific electrical conductivity and wheat sprouting serve as an additional evidence for the hypothesis according to which the EMF-induced water structure changing is an important pathway through which the biological effect of EMF is realized [4].

Most of the theories addressing the mechanism of interaction between biological systems and MFs suggest that the plasma membrane, by virtue of its bioelectrical properties, is the site, where MFs exert their primary effects [5]. To investigate the effect of static magnetic on biological system, it is necessary to clarify interaction mechanisms of magnetic field with biological systems. Simple components of biological system, as cells, biomolecules, artificial membrane, are used in vitro studies that allow reducing of biological variables and more precisely defining and controlling the exposure parameters, compared with in vivo exposure. Erythrocytes are often used as model in exploring the structure and function of the biological cells as well as studying the effect of different physical and chemical factors on the cell. The present study was established to elucidate the changes in biological cells interaction and morphological characterization through in vitro exposure of human erythrocytes to SMF.

\section{MATERIALS AND METHODS}

\subsection{Samples Collection and Preparation}

Twenty blood samples of $5 \mathrm{ml}$ were collected from healthy volunteers of the same age and gender. The blood samples were centrifuged and erythrocytes were separated. Erythrocytes were washed three times in $0.155 \mathrm{M} \mathrm{NaCl}(2,000 \mathrm{rpm}$ for $10 \mathrm{~min})$. After the final centrifugation suspension were diluted to final erythrocytes concentration $2 \%$ in phosphate buffer slain (PBS).

\subsection{SMF System}

The SMF system was constructed in my lab. SMF system is based on the idea of cell tracking velocimetry system. In this system the movement of cells in a well 
defined magnetic field is videotaped [6]. Erythrocytes suspension were placed in a glass sample holder and viewed using an inverted optical microscope in conjunction with a computer linked via digital camera and image capture software. Two rod of iron to produce a magnetic field with large gradient, were placed on both sides of sample holder. The iron rods were alternately magnetized by solenoids controlled by a DC power supply. When magnetized, the magnetic field produced by each rod exerted a force on erythrocytes directed towards the edge of the rod.

\subsection{Erythrocytes Surface Area and Sphericity}

Erythrocytes were suspended in phosphate buffer saline (PBS) at concentration of 5\%. The erythrocytes suspension was placed on the sample holder of SMF system. Image of unexposed erythrocytes (control) was taken then the magnetic field was turned on and the image of exposed erythrocytes was captured. For each value of B images of exposed erythrocytes were compared with control by imaging processing software in order to calculate the changes in erythrocytes surface area $A_{c h}$ and sphericity of each erythrocyte in the image.

The change in erythrocytes surface area was calculated as the following:

$$
\mathrm{A}_{\mathrm{ch}}=\frac{\mathrm{A}_{\mathrm{Ex}}}{\mathrm{A}_{0}}
$$

where $A_{c h}$ is the change in erythrocytes surface area, $A_{E x}$ is the surface area of the erythrocytes after exposed to $\mathrm{SMF}$, and $\mathrm{A}_{0}$ is the surface area of unexposed erythrocytes.

$A_{c h}$ was calculated as the average of 100 erythrocytes for each image. It was taken that if $\mathrm{A}_{\mathrm{ch}}$ greater than one there was an increase in erythrocytes surface area, and if $A_{c h}$ smaller than 1 there was a decrease in erythrocytes surface area.

The sphericity of an erythrocyte is a dimensionless parameter first described by Canham and Burton [7] and is a measure of how spherical a cell is, with values between 0 and 1, for a sphere and laminar disk, respectively. It was calculated as the following:

$$
\text { Sphericity }=4.84 \frac{\mathrm{V}^{2 / 3}}{\mathrm{AEx}}
$$

where $\mathrm{V}$ is the volume of erythrocytes and is taken to be $88.5 \mu \mathrm{m}^{3}$.

\subsection{Erythrocytes Adhesion Number}

Five volume fractions $(0.00001,0.00003,0.00005$, $0.00007,0.00009$ ) of erythrocytes suspensions in PBS were prepared by further dilution of the main erythrocytes suspension. The erythrocytes suspension was placed in sample holder of SMF system. For each sam- ple counting of doublets erythrocytes $\left(\mathrm{N}_{2}\right)$ and singlet erythrocytes $\left(\mathrm{N}_{1}\right)$ was performed. The plot of doublet to singlet ratio versus particle volume fraction should yield a straight line passing through the origin. The gradient of the line is a measure of the adhesion number. The same previous procedure was performed with SMF turned on to calculate the adhesion number of exposed erythrocytes.

\subsection{Statistical Analysis}

The data was present as the mean \pm SD. For each plot, correlation $\left(\mathrm{R}^{2}\right)$ was calculated using Microsoft Excel.

\section{RESULTS}

The erythrocytes surface area increased as the value of magnetic field intensity increased (Figure 1). A strong correlation was found between $\mathrm{A}_{\mathrm{ch}}$ and $\mathrm{B}(\mathrm{mT})\left(\mathrm{R}^{2}=\right.$ 0.95). At low magnetic field the increased in erythrocytes surface area was slightly small. At higher values of magnetic field the increased in erythrocytes surface area raised.

The sphericity of the erythrocytes is inversely proportional to the applied SMF (Figure 2). Under the influence of SMF, the sphercity of erythrocytes was reduced by about $35 \%$ in comparison with unexposed erythrocytes. The decrease in erythrocytes sphericity was reduced rapidly when SMF was applied. A small decrease in sphericity was recorded at higher values of B.

As indicated in Figure 3, The adhesion number increased under the influence of SMF. Acute increase in adhesion number was indicated at $15 \mathrm{mT}$. A strong relation between adhesion number and $\mathrm{B}$ was pointed out $\left(\mathrm{R}^{2}=\right.$ 0.96).

\section{DISCUSSION}

It is generally known that morphological and structural changes to the plasma membrane interfere with many functional and structural features of the cells, leading,

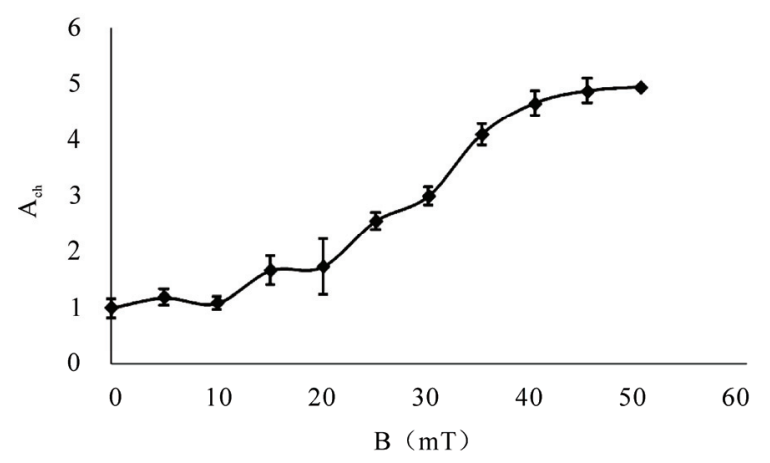

Figure 1. Erythrocytes surface area increased as the values of SMF intensity increased. Strong correlation between $\mathrm{A}_{\mathrm{ch}}$ and $\mathrm{B}$ was observed $\left(R^{2}=0.95\right)$. 


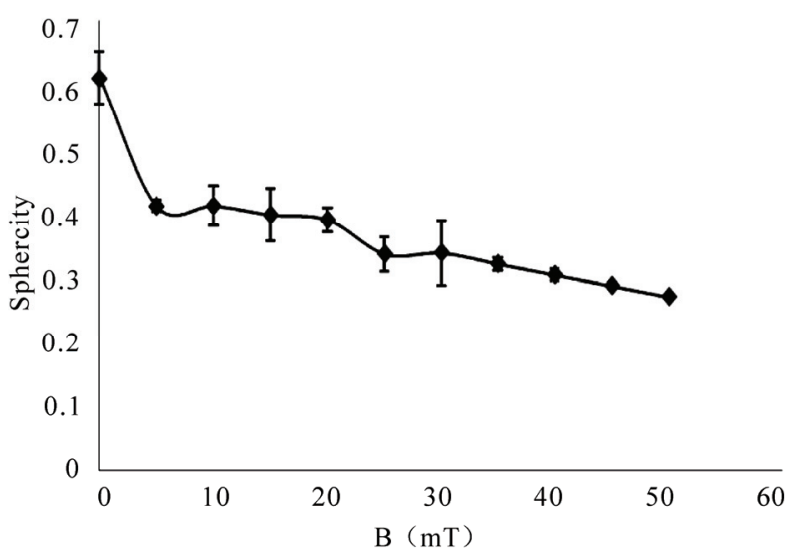

Figure 2. Sphericity of erythrocytes decreased under the influence of SMF. The correlation between sphericity and B is strong $\left(\mathrm{R}^{2}=0.765\right)$

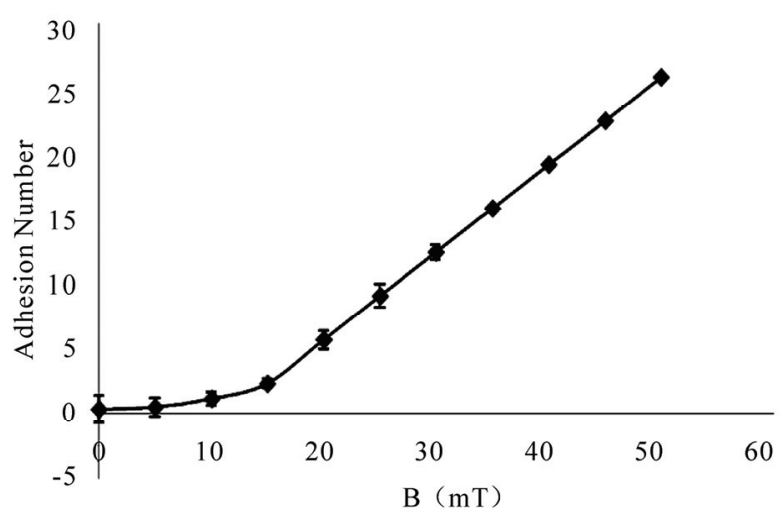

Figure 3. Sharp increase in erythrocytes adhesion number according to increase in SMF intensity (B).

for example, to changes in cellular shape, cytoskeleton arrangement, ion flux, receptor distribution, phagocytosis etc. Modifications in cell shape and plasma membrane as a consequence of exposure to MFs or EMFs have also been reported in many different cells $[8,9,10]$. Moderate-intensity (6 mT) SMFs exert a strong and replicable effect on cell shape and plasma membrane of different cell types [9]. In spite of the fact that this is not a high-intensity magnetic field, it is nevertheless able to produce changes in cell structure and function. Chionna et al. [9] focused their research on cell shape and cell surface modifications, providing evidence for time-related changes. As a general effect, cells growing in suspension lost their round shape and became irregularly elongated, while cells growing attached modified their shape and orientation or detached themselves, becoming freely suspended in the culture medium. Consistent with the previous studies increased in surface area and decreased in sphericity of the erythrocytes were found in the present study. The increased in erythrocytes surface area was at the expense of regular shape of the erythrocytes.

Dilek Ulker Cakir et al. [11] showed that long-term exposure to ELF-EMF decreased the mean volume of thrombocytes in the group of rats exposed for 50 days. Sanjay Jayavanth [12] showed that erythrocytes were monodisperse and with the formation and sedimentation of erythrocytes aggregates. In agreement of the previous studies our results showed that the adhesion of the erythrocytes increase under the influence of SMF compared to control.

From this study it can be concluded that studying the effect of SMF on the microscopic scale give a clear vision about the mechanisms of interaction between living cell and SMF. Also, it could be suggested that the SMF my increase the erythrocytes adhesion hence erythrocytes aggregation. It must be take in a count the changes of erythrocytes morphological structure when dealing with SMF.

\section{REFERENCES}

[1] Higashi, T., Ashida, N. and Takeuchi, T (1997) Orientation of blood cells in static magnetic field. Physica $B$, 237-238, 616-620.

[2] Terumasa, H., Akio, Y., Tetsuya, T. and Muneyuki D. (1995) Effects of static magnetic fields on erythrocyte rheology. Bioelectrochemistry and Bioenergetics, 36, 101-108.

[3] Repacholy, M.H. (2001) Review of health effects and gaps in knowledge. Proceedings of WHO Meeting on EMF Biological Effects and Standard Harmonization in Asia and Okeania, Seoul, 33-42.

[4] Hakobyan, S., Baghdasaryan, N., Amyan, A. and Ayrapetyan, S. (2001) The effect of EMF on water specific electrical conductivity and wheat sprouting. Proceedings of WHO Meeting on EMF Biological Effects and Standard Harmonization in Asia and Okeania, Seoul, 123.

[5] Rosen, A.D. (2003) Mechanism of action of moderate-intensity static magnetic fields on biological systems. Cellular Biochemistry Biophysics, 39, 163-173.

[6] Lee, R.M., Maciej, Z., Masayuki, N., Kara, M., Sigalit, G., Merav, Z., Shlomo, M. and Jeffrey, J.C. (2000) The use of magnetite-doped polymeric microspheres in calibrating cell tracking velocimetry. Journal of Biochemical and Biophysical Methods, 44(1-2), 115-130.

[7] Canham, P.B. and Burton, A.C. (1968) Distribution of size and shape in populations of normal human red cells. Circulation Research, 22, 405-422.

[8] Lisi, A., Pozzi, D., Pasquali, E., Rieti, S., Girasole, M., Cricenti, A., Generosi, R., Serafino, A.L., CongiuCastellano, A., Ravagnan, G., Giuliani, L. and Grimaldi, S. (2000) Three dimensional (3D) analysis of the morphological changes induced by $50 \mathrm{~Hz}$ magnetic field exposure on human lymphoblastoid cells (Raji). Bioelectromagnetics, 21, 46-51.

[9] Chionna, A., Dwikat, M., Panzarini, E., Tenuzzo, B., Carla', E.C., Verri, T., Pagliara, P., Abbro, L. and Dini, L. 
(2003) Cell shape and plasma membrane alterations after static magnetic fields exposure. European Journal of Histochemistry, 47, 299-308.

[10] Rieti, S., Manni, V., Lisi, A., Giuliani, L., Sacco, D., D'Emilia, E., Cricenti, A., Generosi, R., Luce, M. and Grimaldi, S. (2004) SNOM and AFM microscopy techniques to study the effect of non-ionizing radiation on the morphological and biochemical properties of human keratinocytes cell line (HaCaT). Journal of Microscopy, 213, 20-28.
[11] Dilek, U.C., Beran, Y., Mehmet, Z.A., Cemil, S. and Nuriye, M. (2009) Alterations of hematological variations in rats exposed to extremely low frequency magnetic fields $(50 \mathrm{~Hz})$. Archives of Medical Research, 40, 352-356.

[12] Sanjay, J. and Megha, S. (2002) Influence of an inhomogeneous magnetic field on erythrocyte aggregation mechanism an analysis by $\mathrm{He}-\mathrm{Ne}$ laser aggregometer. Journal of Magnetism and Magnetic Materials, 252, 412-414. 